\title{
Real-Time Observation of Exciton-Phonon Coupling Dynamics in Self-Assembled Hybrid Perovskite Quantum Wells
}

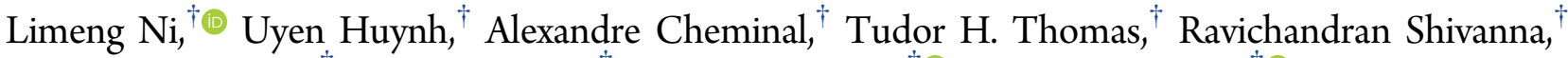
Ture F. Hinrichsen, ${ }^{\dagger}$ Shahab Ahmad, ${ }^{\ddagger}$ Aditya Sadhanala, ${ }^{\dagger}$ and Akshay Rao ${ }^{*}, \dagger$

${ }^{\dagger}$ Cavendish Laboratory, University of Cambridge, JJ Thomson Avenue, Cambridge CB3 OHE, United Kingdom

${ }^{\ddagger}$ Institute for Manufacturing, Department of Engineering, University of Cambridge, 17 Charles Babbage Road, Cambridge CB3 0FS, United Kingdom

\section{Supporting Information}

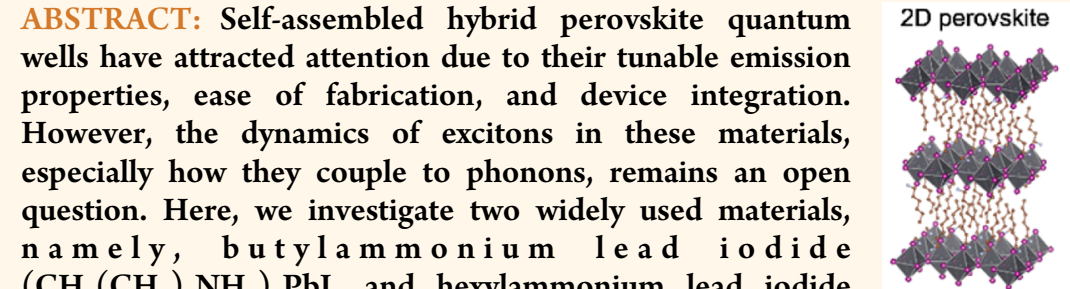
a $\left(\mathrm{CH}_{3}\left(\mathrm{CH}_{2}\right)_{5} \mathrm{NH}_{3} \mathrm{PbI}_{4}\right.$ both of which luminescence tails at room temperature. We performed

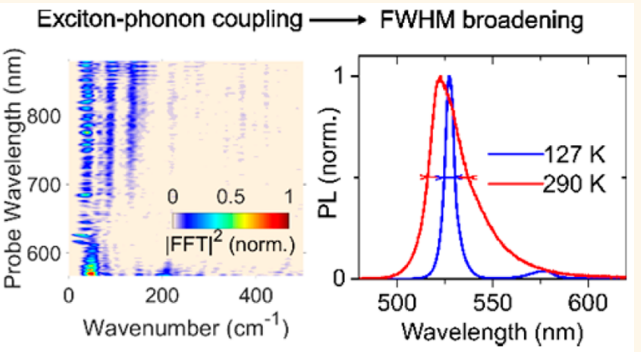
femtosecond vibrational spectroscopy to obtain a real-time picture of the exciton-phonon interaction and directly identified the vibrational modes that couple to excitons. We show that the choice of the organic cation controls which vibrational modes the exciton couples to. In butylammonium lead iodide, excitons dominantly couple to a $100 \mathrm{~cm}^{-1}$ phonon mode, whereas in hexylammonium lead iodide, excitons interact with phonons with frequencies of 88 and 137 $\mathrm{cm}^{-1}$. Using the determined optical phonon energies, we analyzed photoluminescence broadening mechanisms. At low temperatures $(<100 \mathrm{~K})$, the broadening is due to acoustic phonon scattering, whereas at high temperatures, LO phononexciton coupling is the dominant mechanism. Our results help explain the broad photoluminescence line shape observed in hybrid perovskite quantum wells and provide insights into the mechanism of exciton-phonon coupling in these materials.

KEYWORDS: perovskites, 2D materials, temperature-dependent photoluminescence, transient absorption dynamics,

ultrafast vibrational spectroscopy, Raman spectroscopy

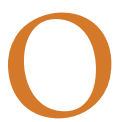
rganometal halide hybrid perovskites have become one of the most promising materials for highly efficient and low-cost optoelectronic devices. The power conversion efficiency of hybrid perovskite photovoltaics has seen a rapid rise in the last 5 years and has exceeded $20 \%{ }^{1}$ In layered two-dimensional (2D) perovskites, corner-sharing metal halide anion $\left[\mathrm{MX}_{4}\right]^{2-}$ quantum wells are confined by aliphatic or aromatic alkylammonium cation barriers (Figure 1a). Due to the hydrophobic property of organic cations, 2D perovskite devices retain their performance under ambient humidity levels. ${ }^{2}$ 2D perovskites have been successfully implemented into solar cell ${ }^{2-4}$ and light-emitting device (LED) fabrication. ${ }^{5}$ Because quantum-confined excitons result in efficient radiative decay, the LEDs exhibit high external quantum efficiency of up to $11.7 \% .^{6}$ These encouraging studies show that hybrid perovskite quantum wells can be a promising candidate for the fabrication of efficient and long-term stable devices.
Electron-phonon coupling in hybrid perovskites is an area that is attracting increasing attention because, for photovoltaic and LED applications, electron-phonon coupling affects charge-carrier mobilities and cooling, and it governs the line shape and line width of the emission. Wright et al. ${ }^{7}$ suggest that electron-phonon coupling at room temperature in $3 \mathrm{D}$ perovskites is mainly due to longitudinal optical (LO) phonon scattering via Fröhlich coupling and that acoustic phonon scattering is negligible. By contrast, Zhu et al. ${ }^{8}$ suggest that large polaron formation in $3 \mathrm{D}$ perovskites protects carriers from optical phonon scattering. Guo et al. ${ }^{9}$ investigated exfoliated 2D perovskite flakes and suggested that acoustic and homopolar optical phonons are the main scattering mechanisms for excitons in these materials. It is thus clear that the role of

Received: June 7, 2017

Accepted: October 24, 2017

Published: October 24, 2017 
a)

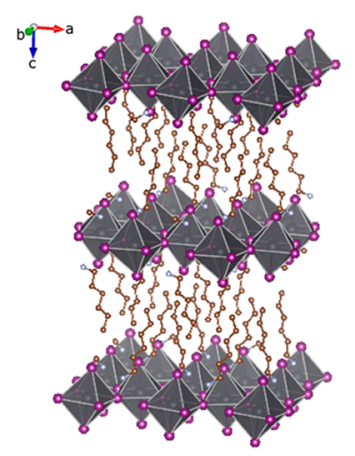

c)

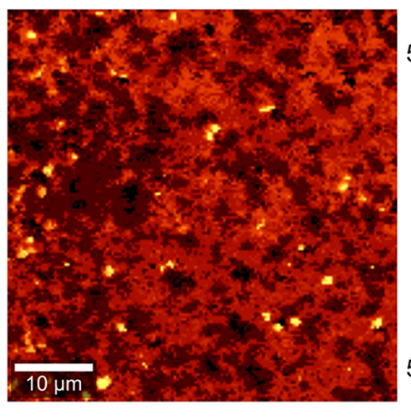

b)

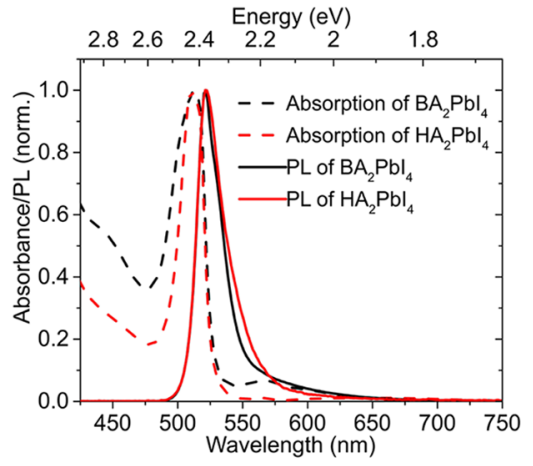

d)
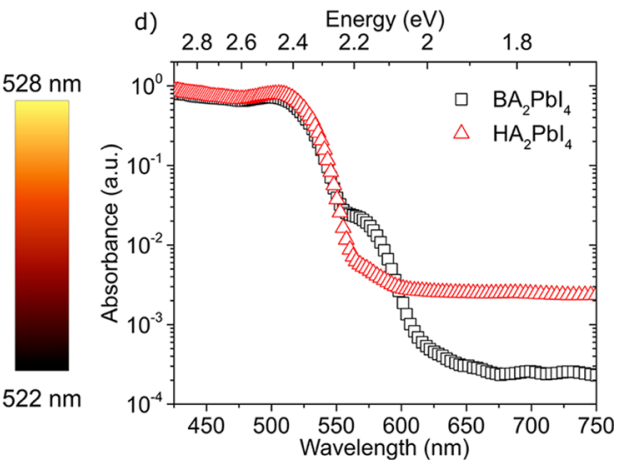

Figure 1. Room temperature crystal structure and optical properties of perovskite quantum wells. (a) Layered crystal structure of $\mathrm{HA}_{2} \mathrm{PbI}_{4}$ (view along the crystal $b$-axis). (b) Normalized absorption and PL spectra of $\mathrm{BA}_{2} \mathrm{PbI}_{4}$ and $\mathrm{HA}_{2} \mathrm{PbI}_{4}$ polycrystalline thin films. (c) Peak position map of a $50 \times 50 \mu \mathrm{m}^{2}$ area on a $\mathrm{BA}_{2} \mathrm{PbI}_{4}$ film under PL microscope. (d) PDS absorption spectra of $\mathrm{BA}_{2} \mathrm{PbI}_{4}$ and $\mathrm{HA}_{2} \mathrm{PbI}_{4}$ thin films.

electron-phonon in hybrid perovskites is still controversial. In addition, many of the widely studied low-dimensional perovskite materials show asymmetric and broad photoluminescence (PL) line shapes ${ }^{4,10-13}$ in comparison to $3 \mathrm{D}$ perovskites that exhibit Gaussian-shaped PL spectra. It is important to understand the nature of the states causing the emission. If they arise due to defects or trap states, it might lower the device performance. However, if it is connected with exciton-phonon coupling, it might provide a means of systematic control via tuning the crystal structure.

Here, we investigate the optical properties of two $2 \mathrm{D}$ perovskite materials, butylammonium lead iodide $\left(\mathrm{CH}_{3}\left(\mathrm{CH}_{2}\right)_{3} \mathrm{NH}_{3}\right)_{2} \mathrm{PbI}_{4}$ and hexylammonium lead iodide $\left(\mathrm{CH}_{3}\left(\mathrm{CH}_{2}\right)_{5} \mathrm{NH}_{3}\right)_{2} \mathrm{PbI}_{4}$, which both show asymmetric $\mathrm{PL}$ shape with a long tail on the low-energy side. We used PL microscopy to investigate whether the asymmetric PL shape is a result of localized inhomogeneity, but no localized defects can be detected at micrometer scale. At low temperatures, the PL of hexylammonium lead iodide becomes narrower and more symmetric, suggesting that the origin of the room temperature asymmetric PL shape is phonon scattering and self-trapped excitons. To directly probe the exciton-phonon coupling and identify the excited-state phonon modes in $2 \mathrm{D}$ perovskites, we used femtosecond transient absorption (fs-TA) spectroscopy. In butylammonium lead iodide, excitons dominantly couple to a phonon mode at $100 \mathrm{~cm}^{-1}$, whereas in hexylammonium lead iodide, excitons couple to phonons with frequencies of 88 and $137 \mathrm{~cm}^{-1}$. Based on the determined phonon modes, the PL broadening mechanism in hexylammonium lead iodide was analyzed using a model that involves acoustic phonons, optical phonons, and impurity scattering. At low temperatures $(<100$ $\mathrm{K})$, the line width broadening is due to acoustic phonon scattering at $0.026 \mathrm{meV} / \mathrm{K}$, whereas at high temperatures, $\mathrm{LO}$ phonon-exciton coupling is the main mechanism. We show that in $2 \mathrm{D}$ perovskites, the coupling strength between $\mathrm{LO}$ phonons and excitons is several tens of meV.

\section{RESULTS AND DISCUSSION}

To study how different organic ligands affect the photophysical properties of $2 \mathrm{D}$ perovskites, spin-coated polycrystalline films of two compounds were investigated: butylammonium lead iodide $\left(\mathrm{CH}_{3}\left(\mathrm{CH}_{2}\right)_{3} \mathrm{NH}_{3}\right)_{2} \mathrm{PbI}_{4}$ and hexylammonium lead iodide $\left(\mathrm{CH}_{3}\left(\mathrm{CH}_{2}\right)_{5} \mathrm{NH}_{3}\right)_{2} \mathrm{PbI}_{4}$. They will be abbreviated as $\mathrm{BA}_{2} \mathrm{PbI}_{4}$ and $\mathrm{HA}_{2} \mathrm{PbI}_{4}$, respectively. Figure $1 \mathrm{~b}$ shows the absorption and PL spectra of thin films of both materials at room temperature. In the absorption spectra, excitonic peaks center at $2.42 \mathrm{eV}(512 \mathrm{~nm}) . \mathrm{BA}_{2} \mathrm{PbI}_{4}$ exhibits an extra absorbing feature between 550 and $600 \mathrm{~nm}$. The photoluminescence spectra of $2 \mathrm{D}$ perovskites peak at $2.37 \mathrm{eV}$ (523 $\mathrm{nm}$ ) with a small Stokes' shift. The PL of both $2 \mathrm{D}$ perovskites is asymmetric and has a broader emission at the low-energy side with a long tail until approximately $675 \mathrm{~nm}$. The observed asymmetric PL shape with a long tail is consistent with previous research. ${ }^{4,10,11}$

The extra absorption feature seen in $\mathrm{BA}_{2} \mathrm{PbI}_{4}$ suggests a more disordered energetic structure or the presence of another phase within the film leading to spatial inhomogeneity. To investigate this further and estimate the energetic disorder, photothermal deflection spectroscopy (PDS) was used as an ultrasensitive absorption measurement. PDS measures the refractive index change due to heat that is caused by nonradiative relaxation when the incoming light is absorbed. The Urbach energy $\left(E_{\mathrm{u}}\right)$ defines the energetic disorder of a material and is given by $A(E)$ $\propto e^{E / E_{u}}$, where $A$ is the absorbance of the material and $E$ the excitation energy. ${ }^{14,15}$ In the PDS spectra (Figure 1d), the absorbance of the extra feature between 550 and $600 \mathrm{~nm}$ is about 10 times higher in $\mathrm{BA}_{2} \mathrm{PbI}_{4}$ than in $\mathrm{HA}_{2} \mathrm{PbI}_{4}$. The Urbach tails in the spectra were fitted with linear regression 
a)

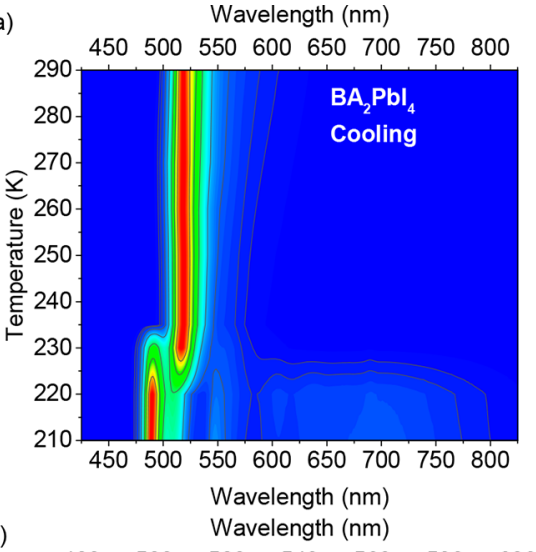

b) Wavelength $(\mathrm{nm})$

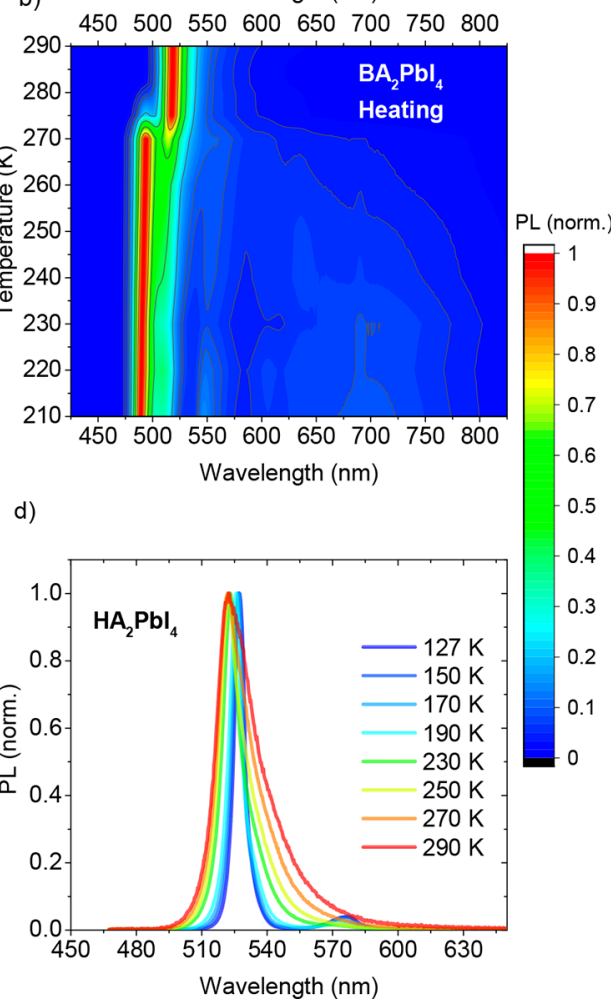

Figure 2. Low-temperature PL measurements on $\mathrm{BA}_{2} \mathrm{PbI}_{4}$ and $\mathrm{HA}_{2} \mathrm{PbI}_{4}$ perovskite films. (a) Temperature-dependent photoluminescence map of $\mathrm{BA}_{2} \mathrm{PbI}_{4}$ obtained by cooling from room temperature. (b) Temperature-dependent photoluminescence map of $\mathrm{BA}_{2} \mathrm{PbI}_{4}$ obtained by heating from $210 \mathrm{~K}$. (c) Temperature-dependent photoluminescence map of $\mathrm{HA}_{2} \mathrm{PbI}_{4}$. (d) Photoluminescence spectra of $\mathrm{HA}_{2} \mathrm{PbI}_{4}$ at different temperatures before phase transition point.

(Supporting Information, SI, Figure S1a). For $\mathrm{BA}_{2} \mathrm{PbI}_{4}$ two Urbach Energies can be defined because it has two absorbing features (SI, Figure S1b). The Urbach energy is calculated to be around 31 and $27 \mathrm{meV}$ for $\mathrm{BA}_{2} \mathrm{PbI}_{4}$ and $\mathrm{HA}_{2} \mathrm{PbI}_{4}$, respectively. These values are about twice the Urbach energy of $3 \mathrm{D}$ perovskite methylammonium lead iodide ${ }^{14}$ but much lower than some organic excitonic system, such as P3HT, whose Urbach energy is about $50 \mathrm{meV} .{ }^{16}$ It is noticeable that below 2 $\mathrm{eV}$ both $\mathrm{BA}_{2} \mathrm{PbI}_{4}$ and $\mathrm{HA}_{2} \mathrm{PbI}_{4}$ exhibit a flat absorbing region, which we consider to arise from sub-band-gap nonradiative states that have been reported previously in 3D perovskites. ${ }^{15,17}$ The different absorbance of these states between the two compounds may be due to different lattice distortion caused by the organic cations (see discussion in SI, Figure S1c).

To further investigate the presence of spatial inhomogeneity, a thin film of $\mathrm{BA}_{2} \mathrm{PbI}_{4}$ was probed with $\mathrm{PL}$ microscopy. The sample was excited by a $405 \mathrm{~nm}$ laser with a beam diameter of about $0.5 \mu \mathrm{m}$. A $50 \times 50 \mu \mathrm{m}^{2}$ area was scanned under the microscope, and the spatially resolved PL map was generated. As seen in Figure 1c, the peak position of the PL varies from 522 to $528 \mathrm{~nm}$ within the scanned area. The full width at halfmaximum (FWHM) changes between 15 and $23 \mathrm{~nm}$, but the shape of the PL spectrum remains the same when probing different spots (SI, Figure S2). This shows that no localized defects can be detected at micrometer scale, and the asymmetric PL shape is not a result of spatial inhomogeneity.

To rule out the possibility that reabsorption may be affecting the emission spectra shape, ${ }^{18}$ we studied the PL of mechanically exfoliated thin crystal flakes of the same material, which are thinner than the spin-coated film. Both the exfoliated flakes and spin-coated film show the same PL (SI, Figure S3), proving that the asymmetric line shape is not due to reabsorption effects. Our finding is consistent with previous research, ${ }^{11}$ where it was shown that thin 2D perovskite crystals with thickness of a few unit cells also exhibit asymmetric PL shape.

Because phonon occupancy drops at low temperature, ${ }^{19}$ temperature-dependent photoluminescence spectra were recorded to study the effect of phonon scattering on the PL shape. Figure 2a,b shows the steady-state temperature-dependent $\mathrm{PL}$ map of a $\mathrm{BA}_{2} \mathrm{PbI}_{4}$ thin film excited with a continuous wave (CW) $405 \mathrm{~nm}$ laser and measured in $10 \mathrm{~K}$ steps. At low temperature, the PL peak shifts from 520 to $493 \mathrm{~nm}$ due to an orthorhombic-to-orthorhombic phase transition. ${ }^{20}$ The impact of structural phase changes of $\left(\mathrm{C}_{n} \mathrm{H}_{2 n+1} \mathrm{NH}_{3}\right)_{2} \mathrm{PbI}_{4}$ compounds on their optical properties was first reported by Ishihara et al. ${ }^{21}$ In addition, we observed a hysteresis loop when measuring the temperature-dependent PL spectrum, meaning that the phase transition occurs at $230 \mathrm{~K}$ when cooling down (Figure 2a) but at approximately $280 \mathrm{~K}$ when heating up (Figure $2 \mathrm{~b}$ ). The hysteresis effect of $\mathrm{BA}_{2} \mathrm{PbI}_{4}$ shows that a metastable state exists between 230 and $280 \mathrm{~K}$, and the crystal structure is switchable within this temperature range. Temperature-dependent UV-vis spectroscopy further confirmed the hysteresis of phase transition (SI, Figure S4). Thermal hysteresis of $\mathrm{BA}_{2} \mathrm{PbI}_{4}$ was also revealed in previous research using calorimetry. ${ }^{20} \mathrm{~A}$ similar hysteresis loop of phase transition was observed on $\left(\mathrm{C}_{12} \mathrm{H}_{25} \mathrm{NH}_{3}\right)_{2} \mathrm{PbI}_{4} 2 \mathrm{D}$ perovskite crystals ${ }^{22}$ and on $3 \mathrm{D}$ perovskite thin films.

It is also notable that in both Figure $2 \mathrm{a}, \mathrm{b}$ the low-energy emission from $550 \mathrm{~nm}$ onward starts to become more pronounced when the phase transition happens. This indicates that the long tail at the low-energy side of the PL emission in 

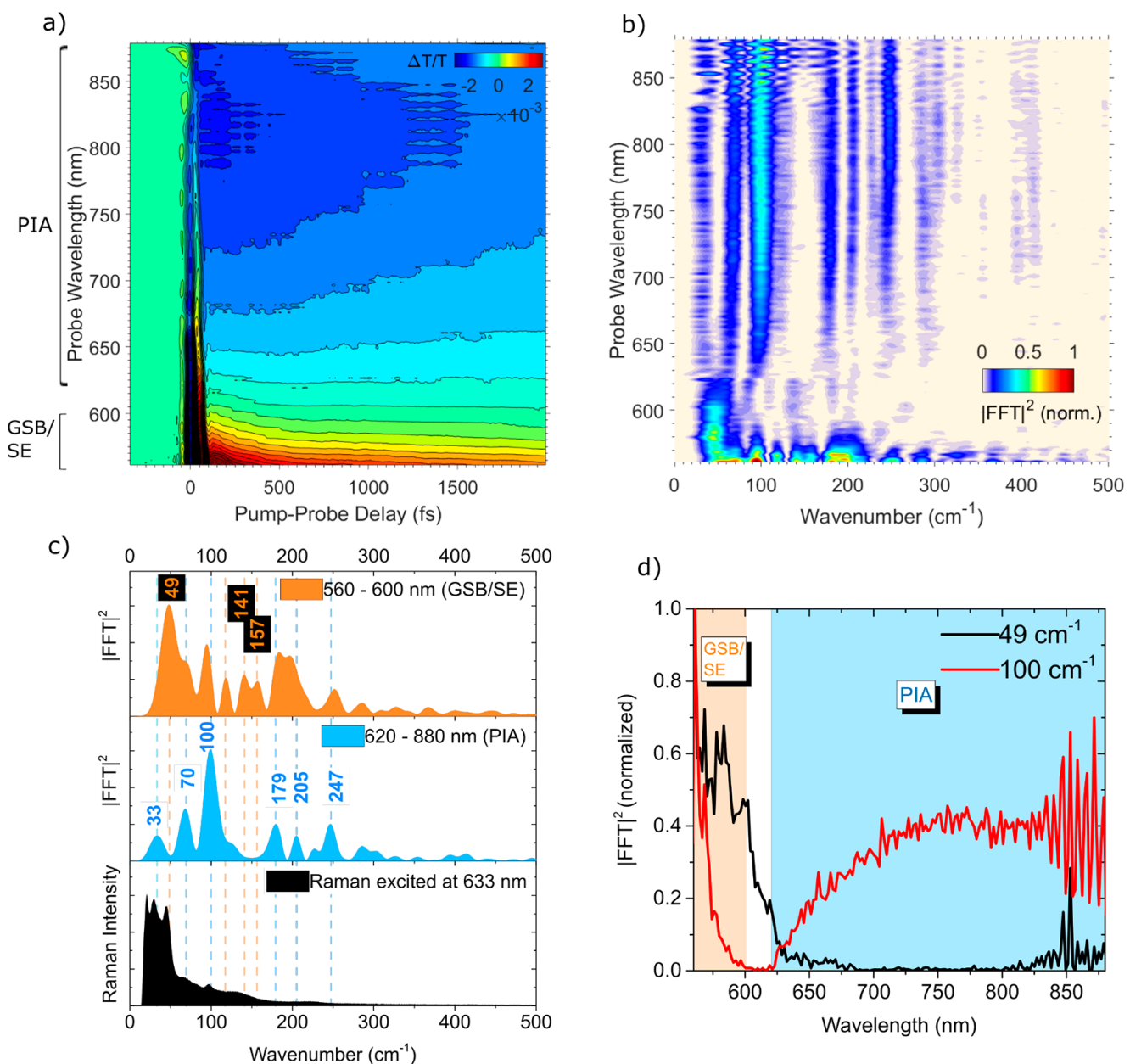

Figure 3. Femtosecond transient absorption on a $\mathrm{BA}_{2} \mathrm{PbI}_{4}$ perovskite film. (a) Transient absorption map of $\mathrm{BA}_{2} \mathrm{PbI}_{4}$ pumped at $520 \mathrm{~nm}$. (b) Wavelength-resolved Fourier transform power map of $\mathrm{BA}_{2} \mathrm{PbI}_{4}$. (c) Fourier transformed oscillation spectra of $\mathrm{BA}_{2} \mathrm{PbI}_{4}$ averaged over $560-$ $600 \mathrm{~nm}(\mathrm{GSB} / \mathrm{SE})$ and $620-880 \mathrm{~nm}$ (PIA) in comparison with Raman spectrum excited at $633 \mathrm{~nm}$. The three black highlighted modes are the modes found in the GSB/SE but are absent in the PIA. (d) Normalized Fourier transform power of 49 and $100 \mathrm{~cm}^{-1}$ vibrational modes in $\mathrm{BA}_{2} \mathrm{PbI}_{4}$ throughout the probe wavelengths.

Figure 1 probably results from states related to a different phase or lattice distortion. The fact that inhomogeneity is not detected in the PL microscopy studies means that these different phases or lattice distortions are presumably mixed on sub-micrometer scales and uniformly distributed in the film. Further experiments, such as micro-X-ray diffraction or nearfield scanning optical microscopy, need to be conducted to examine this sub-micrometer-scale inhomogeneity.

Temperature-dependent $\mathrm{PL}$ of $\mathrm{HA}_{2} \mathrm{PbI}_{4}$ behaves completely different from that of $\mathrm{BA}_{2} \mathrm{PbI}_{4}$ (Figure $2 \mathrm{c}$ ). The phase transition happens at a much lower temperature than $\mathrm{BA}_{2} \mathrm{PbI}_{4}$. The emission of the low-temperature phase of $\mathrm{HA}_{2} \mathrm{PbI}_{4}$ peaks at 533 $\mathrm{nm}(2.33 \mathrm{eV})$, which is red-shifted in comparison to the emission of the room temperature phase. Because the emission peaks of the two phases of $\mathrm{HA}_{2} \mathrm{PbI}_{4}$ are close to each other, it is hard to determine the exact phase transition point. By plotting the separate contribution of the two phases over the total integrated PL intensity versus temperature (SI, Figure S5), it is found that the PL intensity of the high-energy phase drops and the intensity of the low-energy phase rises when the temperature is below $127 \mathrm{~K}$, suggesting the phase transition point is at approximately $127 \mathrm{~K}$. Billing et al. suggested that at low temperature, $\mathrm{HA}_{2} \mathrm{PbI}_{4}$ undergoes a orthorhombic-tomonoclinic phase transition. ${ }^{20}$ Furthermore, through temper- ature-dependent UV-vis spectroscopy, no hysteresis effect was observed for $\mathrm{HA}_{2} \mathrm{PbI}_{4}$ (SI, Figure S6). The fact that the temperature-dependent $\mathrm{PL}$ is different between $\mathrm{BA}_{2} \mathrm{PbI}_{4}$ and $\mathrm{HA}_{2} \mathrm{PbI}_{4}$ suggests that the organic ligands have a controlling role on the crystal structure of the quantum wells.

In order to see how the PL line shape changes with temperature before the phase transition happens, normalized $\mathrm{PL}$ spectra of $\mathrm{HA}_{2} \mathrm{PbI}_{4}$ at different temperatures above $127 \mathrm{~K}$ are shown in Figure 2d. At a temperature of $150 \mathrm{~K}$ and lower, the PL spectrum consists of a symmetric peak (510-545 nm) and broad side emission $(560-590 \mathrm{~nm})$. The symmetric part of the PL can be assigned to the emission of free excitons. As discussed before in the case of $\mathrm{BA}_{2} \mathrm{PbI}_{4}$, the emission between 560 and $590 \mathrm{~nm}$ may associate with a different phase or lattice distortion. Remarkably, asymmetric broadening is observed with increasing temperature, where the broadening of the lowenergy part of the emission (from peak to $550 \mathrm{~nm}$ ) is stronger than that on the high-energy side (wavelengths shorter than peak). This can be interpreted as the formation of self-trapped excitons below the optical band gap due to strong excitonphonon coupling. ${ }^{10,12,13,24}$ Thus, the PL line shape of $2 \mathrm{D}$ perovskites is controlled by the presence of lattice distortion and exciton-phonon interactions. At low temperature, where these interactions are suppressed, the line shape is symmetric. 

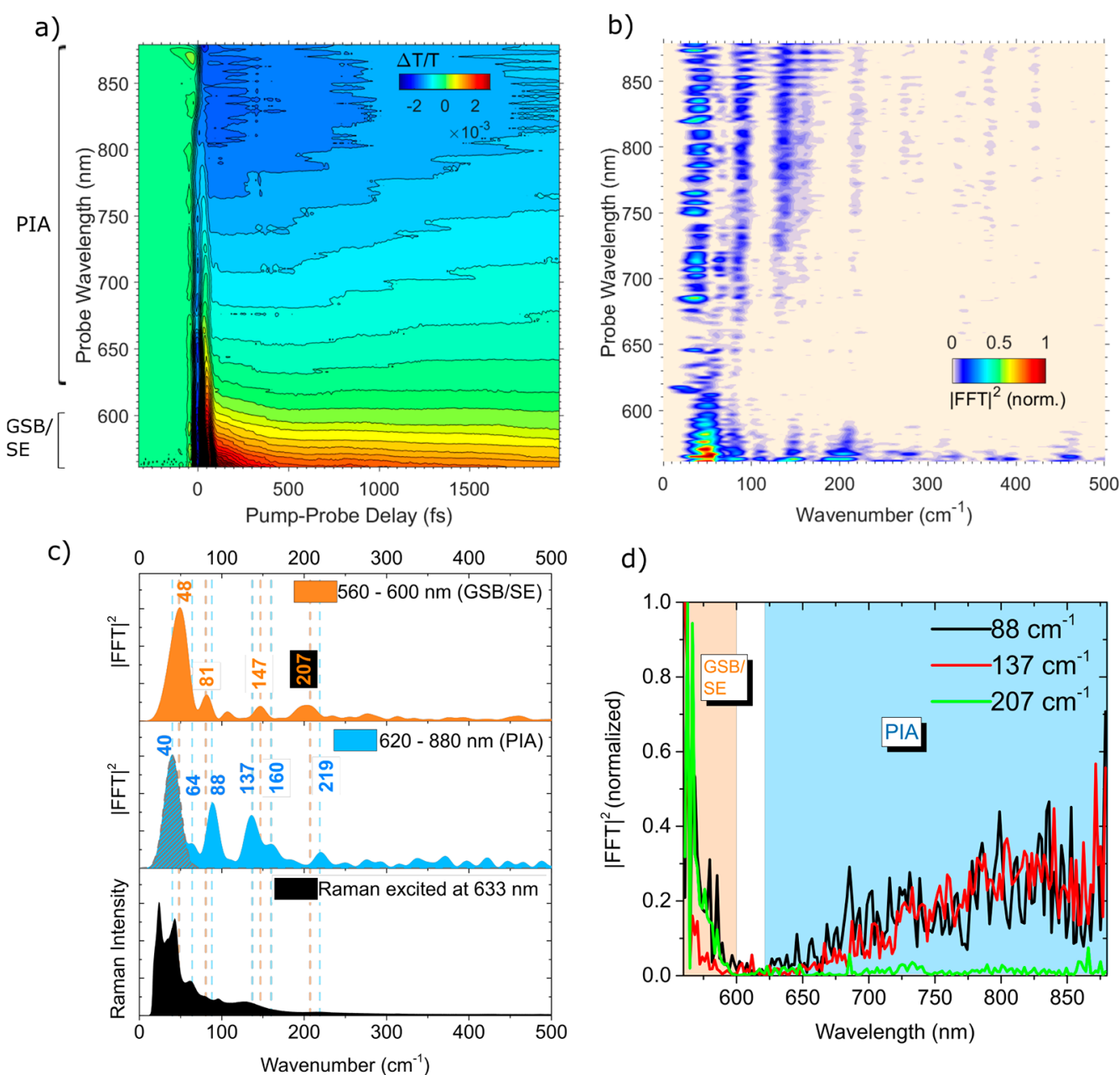

Figure 4. Femtosecond transient absorption on a $\mathrm{HA}_{2} \mathrm{PbI}_{4}$ perovskite film. (a) Transient absorption map of $\mathrm{HA}_{2} \mathrm{PbI}_{4}$ pumped at $520 \mathrm{~nm}$. (b) Wavelength-resolved Fourier transform power map of $\mathrm{HA}_{2} \mathrm{PbI}_{4}$. (c) Fourier transformed oscillation spectra of $\mathrm{HA}_{2} \mathrm{PbI}_{4}$ averaged over 560$600 \mathrm{~nm}(\mathrm{GSB} / \mathrm{SE})$ and 620-880 $\mathrm{nm}$ (PIA) in comparison with Raman spectrum excited at $633 \mathrm{~nm}$. The black highlighted mode is a mode found in the GSB/SE but absent in the PIA. (d) Normalized Fourier transform power of 88, 137, and $207 \mathrm{~cm}^{-1}$ vibrational modes in $\mathrm{HA}_{2} \mathrm{PbI}_{4}$ throughout the probe wavelengths.

At room temperature, asymmetric broadening occurs due to strong exciton-phonon coupling. Interestingly, between 150 and $250 \mathrm{~K}$, the PL peak position and the optical band gap are blue-shifted at $0.24 \mathrm{meV} / \mathrm{K}$ with increasing temperature (SI, Figure S7). As has been reported in a previous study, ${ }^{11}$ the slight increase of the optical band gap may relate to lattice expansion.

Although electron-phonon coupling in hybrid perovskite materials has been studied before, ${ }^{7,9,25,26}$ no experiments have provided a real-time picture of how excitons couple to phonon modes. It is crucial to identify which modes dominate the coupling because it can help us understand the mechanism of exciton-phonon coupling in perovskites. For instance, based on the phonon energy, we can distinguish between acoustic phonons and optical phonons. In perovskites, the acoustic phonons normally have much lower energy than optical phonons. $^{7}$ Moreover, we will use the identified phonon modes to evaluate the PL broadening mechanisms later. We therefore conducted fs-TA spectroscopy to directly probe the excited-state and ground-state phonon vibrational modes in $2 \mathrm{D}$ perovskites. Spin-coated films of $\mathrm{BA}_{2} \mathrm{PbI}_{4}$ and $\mathrm{HA}_{2} \mathrm{PbI}_{4}$ were excited with a 16 fs pump pulse resonant with the optical band gap $(520 \mathrm{~nm})$. They were probed with a chirped broad-band white light continuum pulse $(560-880 \mathrm{~nm})$. Figure 3a shows a transient absorption map of $\mathrm{BA}_{2} \mathrm{PbI}_{4}$, where $\Delta T / T$ is plotted as functions of probe wavelength and time delay between pump and probe. $\Delta T$ is the change in the transmission of the sample with/without the pump pulse, and $T$ is the transmission without the pump pulse. The positive signal from $560 \mathrm{~nm}$ to around $600 \mathrm{~nm}$ represents the ground-state bleach (GSB) and stimulated emission (SE), which agrees with steady-state PL and absorption spectra and is consistent with previously reported transient absorption measurements probed from 450 to $750 \mathrm{~nm}$ by Wu et al. ${ }^{10}$ The negative signal between 620 and $880 \mathrm{~nm}$ corresponds to photoinduced absorption (PIA), that is, the absorption of excited states generated by the pump pulse.

The use of a compressed $16 \mathrm{fs}$ pump pulse generates wave packets both in the ground and excited states. ${ }^{27,28}$ It allows us to access not only the electronic dynamics of the photoexcitation but also the molecular vibrational modes due to the generation of wave packets. In order to obtain these vibrational dynamics, we globally fitted the map in Figure 3a with a linear combination of three exponential functions that describes the electronic decay dynamics (SI, Figure S8a). Subtracting these electronic dynamics leaves the residual vibrational oscillations (SI, Figure S8b), which are then fast Fourier transformed into 
the frequency domain. Only data after $160 \mathrm{fs}$ were considered in order to exclude the coherent artifact contributions. The resolution of fast Fourier transform (FFT) is around $17 \mathrm{~cm}^{-1}$, and the lowest frequency that can be resolved is about $33 \mathrm{~cm}^{-1}$, which is limited by the probe time window of 2 ps. The Fourier transformed result of $\mathrm{BA}_{2} \mathrm{PbI}_{4}$ is shown as a wavelengthresolved Fourier transform power map in Figure $3 \mathrm{~b}$, which identifies which TA features the vibrational modes modulate. It can be clearly seen that vibrational modes are present in the GSB/SE as well as in the PIA region. The oscillation frequency is shown until $500 \mathrm{~cm}^{-1}$, as no higher frequency mode was identified.

It is necessary to distinguish between ground-state and excited-state phonons because only the excited-state phonons couple to excitons and modulate the exciton relaxation pathway. They can therefore have influence on the PL line width via exciton-phonon coupling. Thus, we compared the vibrational modes that are found in the Fourier transformed fsTA data with the ground-state phonon modes in the steadystate Raman spectrum, where the sample was excited offresonantly at $633 \mathrm{~nm}$. In Figure 3c, the vibrational modes from Figure $3 \mathrm{~b}$ were averaged over GSB/SE (560-600 nm) and PIA $(620-880 \mathrm{~nm})$ because the modes in the GSB are ground-state phonons and in the SE and PIA are excited-state phonons. Unfortunately, due to the small Stokes' shift of the materials and the limitation of the probe wavelength, it is difficult to distinguish between GSB and SE. In the GSB/SE region, we identified three vibrational modes (marked in black) with frequencies of 49,141 , and $157 \mathrm{~cm}^{-1}$ absent in the PIA region. No previous experimental or theoretical work on $2 \mathrm{D}$ perovskite quantum wells assigns these modes. Despite this, we consider that $2 \mathrm{D}$ and $3 \mathrm{D}$ perovskites should share some common vibrational modes as both consist of $\mathrm{PbI}_{6}$ octahedra. PerezOsorio et al. ${ }^{29}$ calculated the vibrational modes in $3 \mathrm{D}$ perovskite $\mathrm{MAPbI}_{3}$. They assign the modes below $40 \mathrm{~cm}^{-1}$, between 40 and $50 \mathrm{~cm}^{-1}$ and between 50 and $100 \mathrm{~cm}^{-1}$ to $\mathrm{Pb}-$ $\mathrm{I}-\mathrm{Pb}$ rocking motion, bending motion, and $\mathrm{Pb}-\mathrm{I}$ stretching motion, respectively. The frequencies between 120 and 180 $\mathrm{cm}^{-1}$ are assigned to spin, libration, and translation of MA cations. $^{29}$ We expect the motion of the cations and their corresponding frequencies would be different between $2 \mathrm{D}$ and $3 \mathrm{D}$ perovskites, as the cations in $2 \mathrm{D}$ perovskites are larger than MA cations, and they are located between the $\mathrm{PbI}_{6}$ octahedra sheets, whereas in $3 \mathrm{D}$ perovskites, the cations are confined in the octahedra holes. In the PIA region, the $100 \mathrm{~cm}^{-1}$ mode seems to stand out among the observed vibrational modes. In general, comparing the Fourier transformed TA spectra with the off-resonant Raman spectrum, the former gives better peak resolution than the broad Raman spectrum, which shows only diffuse and continuum modes with frequencies lower than 200 $\mathrm{cm}^{-1}$. This comparison reveals the benefit of our ultrafast timedomain approach.

In Figure 3d, the normalized $|\mathrm{FFT}|^{2}$ of the dominant mode in the GSB/SE $\left(49 \mathrm{~cm}^{-1}\right)$ and PIA $\left(100 \mathrm{~cm}^{-1}\right)$ is plotted against the probe wavelength. It can be clearly seen that the $49 \mathrm{~cm}^{-1}$ mode is in the GSB and in the off-resonant Raman spectrum but not in the PIA, suggesting a ground-state phonon mode. The $100 \mathrm{~cm}^{-1}$ mode is in the GSB/SE, in the off-resonant Raman spectrum, and in the PIA, meaning that it is a mode that exists in excited states as well as in the ground state.

The phonon modes of $\mathrm{HA}_{2} \mathrm{PbI}_{4}$ were also investigated. Figure $4 \mathrm{a}$ shows a transient absorption map of $\mathrm{HA}_{2} \mathrm{PbI}_{4}$. The $\mathrm{GSB} / \mathrm{SE}$ and PIA wavelengths are similar to those of $\mathrm{BA}_{2} \mathrm{PbI}_{4}$.
However, as shown in Figure $4 \mathrm{~b}$, the wavelength-resolved Fourier transform power map of $\mathrm{HA}_{2} \mathrm{PbI}_{4}$ is different from the map of $\mathrm{BA}_{2} \mathrm{PbI}_{4}$ (Figure $3 \mathrm{~b}$ ), suggesting that changing the organic cations in $2 \mathrm{D}$ perovskite will affect its phonon modes. The vibrational modes were averaged over GSB/SE (560-600 $\mathrm{nm})$ and PIA (620-880 $\mathrm{nm}$ ) in Figure 4c and were compared with the off-resonant Raman spectrum. In the GSB/SE region, a black marked vibrational mode with a frequency of $207 \mathrm{~cm}^{-1}$ is absent in the PIA. In Figure 4d, the normalized $|F F T|^{2}$ of the three dominant vibrational modes in the GSB/SE and PIA (88, 137 , and $207 \mathrm{~cm}^{-1}$ ) is plotted against the probe wavelength. We disregarded the $40 \mathrm{~cm}^{-1}$ mode (gray shadowed) in the PIA because the large $|F F T|^{2}$ amplitude of this mode is due to the high noise level, as it is close to the low-frequency cutoff of the Fourier transform window. The $207 \mathrm{~cm}^{-1}$ mode is in the GSB, but absent in the PIA, suggesting a ground-state phonon mode. The 88 and $137 \mathrm{~cm}^{-1}$ modes are in the GSB/SE, in the PIA and in the off-resonant Raman spectrum, which means that they are phonon modes that exist both in the ground state and in the excited states.

As control experiments, we measured blank substrates and noncrystallized $\mathrm{PbI}_{2}$ precursor. In the blank substrate, no vibrational mode is observed (SI, Figure S9). In the $\mathrm{PbI}_{2}$ precursor, only one dominant mode at $108 \mathrm{~cm}^{-1}$ exists (SI, Figure S10). We therefore confirm that the multiple phonon modes obtained above belong to $\mathrm{BA}_{2} \mathrm{PbI}_{4}$ and $\mathrm{HA}_{2} \mathrm{PbI}_{4}$ materials.

To sum up, using fs-TA spectroscopy, we directly observed the exciton-phonon coupling dynamics in $2 \mathrm{D}$ perovskite. As we aim at understanding the PL line width broadening through phonon scattering, we focus on the assigned excited-state phonon modes and disregard ground-state phonon modes. Comparing the phonon modes in $\mathrm{BA}_{2} \mathrm{PbI}_{4}$ and $\mathrm{HA}_{2} \mathrm{PbI}_{4}$, multiple phonon modes exist in $2 \mathrm{D}$ perovskites at frequencies lower than $300 \mathrm{~cm}^{-1}$ in both compounds. Modes around 45, 70 , and $100 \mathrm{~cm}^{-1}$ are found in both $\mathrm{BA}_{2} \mathrm{PbI}_{4}$ and $\mathrm{HA}_{2} \mathrm{PbI}_{4}$. However, some modes are ligand-dependent; for instance, the modes around 33 and $250 \mathrm{~cm}^{-1}$ are clearer in $\mathrm{BA}_{2} \mathrm{PbI}_{4}$ but not obvious in $\mathrm{HA}_{2} \mathrm{PbI}_{4}$. Table 1 summarizes the dominant phonon

Table 1. Dominant Ground-State and Excited-State Phonon Modes in $\mathrm{BA}_{2} \mathrm{PbI}_{4}$ and $\mathrm{HA}_{2} \mathrm{PbI}_{4}$ Identified by Femtosecond Transient Absorption Spectroscopy

\begin{tabular}{lcc} 
& $\begin{array}{c}\text { phonon modes in } \\
\mathrm{BA}_{2} \mathrm{PbI}_{4}\left(\mathrm{~cm}^{-1}\right)\end{array}$ & $\begin{array}{c}\text { phonon modes in } \\
\mathrm{HA}_{2} \mathrm{PbI}_{4}\left(\mathrm{~cm}^{-1}\right)\end{array}$ \\
$\begin{array}{lcc}\text { only in the ground state } \\
\begin{array}{c}\text { in both the ground state and } \\
\text { excited states }\end{array}\end{array}$ & 49 & 207 \\
\hline
\end{tabular}

modes in the ground state and excited states of both compounds. In $\mathrm{BA}_{2} \mathrm{PbI}_{4}$, excitons dominantly couple to a mode at $100 \mathrm{~cm}^{-1}(12.4 \mathrm{meV})$, whereas in $\mathrm{HA}_{2} \mathrm{PbI}_{4}$, excitons mainly interact with $88 \mathrm{~cm}^{-1}(10.9 \mathrm{meV})$ and $137 \mathrm{~cm}^{-1}(17.0$ $\mathrm{meV}$ ) modes. The phonon energies of $\mathrm{BA}_{2} \mathrm{PbI}_{4}$ and $\mathrm{HA}_{2} \mathrm{PbI}_{4}$ are similar to the calculated values of $3 \mathrm{D}$ perovskites ${ }^{7,29}$ and another compound of $2 \mathrm{D}$ perovskite (PEPI). ${ }^{25}$ These modes are attributed to optical phonons because acoustic phonons in perovskite materials have much lower energy $(<2.5 \mathrm{meV})$. Crucially, these results show that the dominant mode to which the excitons couple is related to the organic cations. This means that the exciton-phonon coupling and properties affected by it, such as the PL line shape, can be controlled by the choice of organic cation. 
Using the phonon energies that were measured by fs-TA spectroscopy, we can qualitatively study PL broadening mechanisms as a result of exciton-phonon coupling. We extracted the FWHM of the PL spectra of $\mathrm{HA}_{2} \mathrm{PbI}_{4}$ in Figure $2 \mathrm{c}$ as a function of temperature. Because a phase transition occurs at low temperature, the two phases are separated by fitting the spectra into two Gaussian peaks (SI, Figure S11). In Figure 5, the FWHM of the high-energy peak, which is the dominant phase at room temperature, is plotted over temperature. The relationship between the PL line width and phonon scattering in most inorganic materials can be estimated by the following equation: ${ }^{30-32}$

$$
\Gamma(T)=\Gamma_{0}+\Gamma_{\mathrm{ac}} T+\frac{\Gamma_{\mathrm{LO}}}{e^{E_{\mathrm{LO}} / k T}-1}+\Gamma_{\mathrm{imp}} e^{-E_{\mathrm{b}} / k T}
$$

The first term $\Gamma_{0}$ is the inhomogeneous broadening term and the line width at $0 \mathrm{~K}$. The second term describes the homogeneous broadening due to the acoustic phonon scattering via deformation and piezoelectric potentials, ${ }^{30}$ where $\Gamma_{\mathrm{ac}}$ is the coupling strength between exciton and acoustic phonons. The third term corresponds to the homogeneous broadening that results from LO phonon (Fröhlich) scattering with exciton and optical phonon coupling strength of $\Gamma_{\mathrm{LO}}$ and the energy of $\mathrm{LO}$ phonon of $E_{\mathrm{LO}}$. The last term describes the inhomogeneous broadening caused by ionized impurities. When impurities are fully ionized, $\Gamma_{\text {imp }}$ is their contribution to the line width broadening and $E_{\mathrm{b}}$ is the average binding energy of impurities. ${ }^{30,32}$ However, this approach has some limitation. For instance, it is assumed that the phonon energy is independent of temperature, but $2 \mathrm{D}$ perovskite quantum wells are soft materials and their phonon energy could possibly change with temperature. Another limitation is that the model only includes a single LO phonon mode, although the fs-TA spectroscopy has shown that multiple modes can couple to excitons in $2 \mathrm{D}$ perovskites. It is an open question whether the line width broadening is a linear combination of the coupling between excitons and different LO phonons. Nevertheless, this model is a good tool to help understand the mechanisms of exciton-phonon coupling in perovskite materials.

The optical phonons that couple to excited states were determined by fs-TA spectroscopy (Table 1). $E_{\mathrm{LO}}$ is found to be about $12.4 \mathrm{meV}\left(100 \mathrm{~cm}^{-1}\right)$ for $\mathrm{BA}_{2} \mathrm{PbI}_{4}$ and $10.9 \mathrm{meV}(88$ $\left.\mathrm{cm}^{-1}\right)$ and $17.0 \mathrm{meV}\left(137 \mathrm{~cm}^{-1}\right)$ for $\mathrm{HA}_{2} \mathrm{PbI}_{4}$. By fitting the curve of the integrated $\mathrm{PL}$ of the high-energy emission of $\mathrm{HA}_{2} \mathrm{PbI}_{4}$ over temperature using the Arrhenius formula, ${ }^{33-35}$ the binding energy of the ionized impurities $E_{\mathrm{b}}$ is calculated to be about $67 \mathrm{meV}$ (SI, Figure S12), which is consistent with the values previously reported for another $2 \mathrm{D}$ perovskite, namely, $\left(\mathrm{C}_{6} \mathrm{H}_{5} \mathrm{C}_{2} \mathrm{H}_{4} \mathrm{NH}_{3}\right)_{2} \mathrm{PbI}_{4}$ (abbreviated as PEPI). ${ }^{25}$ We note that the fitting of the impurity binding energy has some limitation because it is possible that the monomolecular recombination rate constant and the radiative efficiency of $2 \mathrm{D}$ perovskites could be temperature-dependent. The determined $E_{\mathrm{LO}}$ and $E_{\mathrm{b}}$ are inserted into eq 1 , and the curve of FWHM over temperature is fitted in Figure 5.

In polar semiconductors, electrons at low temperature $(<100$ $\mathrm{K})$ have low energy and cannot emit optical phonons with energy of tens of meV. At room temperature, optical phonons can be sufficiently emitted by high-energy electrons. In addition, due to high thermal occupation number of optical phonons, the probability of electrons absorbing optical phonons is high. Consequently, optical phonons dominate

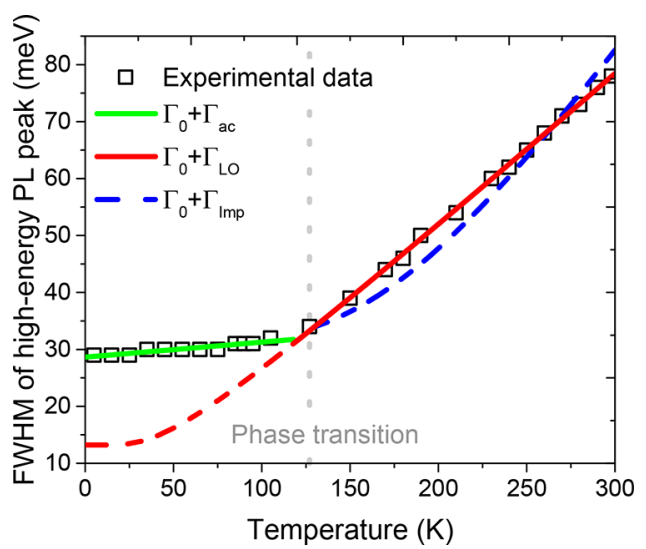

Figure 5. Temperature-dependent PL FWHM of the high-energy phase of $\mathrm{HA}_{2} \mathrm{PbI}_{4}$ and fitting examples according to eq 1 . The green line is the combination of inhomogeneous broadening and acoustic phonon. The red curve shows the effect of inhomogeneous broadening and LO phonon $\left(E_{\mathrm{LO}}=10.9 \mathrm{meV}\right)$ coupling to excitons. The red dashed curve is the extrapolation of the red solid curve to $0 \mathrm{~K}$. The blue dashed curve is the fitting of inhomogeneous broadening and impurity $\left(E_{\mathrm{b}}=67 \mathrm{meV}\right)$ scattering. The gray dotted line shows the phase transition point.

over acoustic phonons at room temperature. ${ }^{19}$ Therefore, we fitted the low-temperature regime with inhomogeneous broadening at $0 \mathrm{~K}\left(\Gamma_{0}\right)$ and acoustic phonons scattering $\left(\Gamma_{\mathrm{ac}}\right)$ (green curve). $\Gamma_{\mathrm{ac}}$ is equal to $0.026 \pm 0.003 \mathrm{meV} / \mathrm{K}$, which is consistent with the value for another $2 \mathrm{D}$ perovskite PEPI $(0.03$ $\pm 0.01 \mathrm{meV} / \mathrm{K})^{25}$ and 1 order of magnitude larger than the value for GaAs quantum wells. ${ }^{36}$ The high-temperature regime $(T>100 \mathrm{~K})$ was fitted using the combination of $\Gamma_{0}$ and LO phonon-exciton coupling $\left(\Gamma_{\mathrm{LO}}\right)$ (red curve). The fitting parameters of each curve are listed in Table 2. The red curve

Table 2. Parameters of the Best Fit. (*Fixed Values That Were Determined by fs-TA Spectroscopy)

\begin{tabular}{cccc} 
& $\Gamma_{0}(\mathrm{meV})$ & $E_{\mathrm{LO}} *(\mathrm{meV})$ & $\Gamma_{\mathrm{LO}}(\mathrm{meV})$ \\
LO phonon-exciton coupling & $13.2 \pm 0.4$ & 10.9 & $34.2 \pm 0.3$ \\
& $18.7 \pm 0.3$ & 17 & $55.8 \pm 0.4$ \\
\hline
\end{tabular}

is also extrapolated into the low-temperature regime (dashed line). The model does not match the experimental data in this regime. There could be two explanations. One reason is that, as already mentioned, at low temperatures the population of optical phonons is low. Another reason is that due to the phase transition of the material below $127 \mathrm{~K}$, the phonon energy and behavior may change due to lattice distortion. Similar phenomenon was observed for $3 \mathrm{D}$ perovskites $\left(\mathrm{MAPbI}_{3}\right.$ and $\left.\mathrm{MAPbBr}{ }_{3}\right){ }^{7}$ As seen in the blue dashed curve, we also tried the fitting at the high-temperature regime using $\Gamma_{\mathrm{LO}}$ and impurity scattering $\left(\Gamma_{\operatorname{Imp}}\right)$. It does not contribute much to the line width broadening because the shape of the fitted curve does not agree with the experimental data. In previous study on electronphonon coupling in $3 \mathrm{D}$ perovskite, it was also found that scattering with ionized impurities does not play any major role.

The coupling strengths between excitons and two different LO phonons are around 30 and $60 \mathrm{meV}$. These values are similar to the LO phonon-electron coupling strength in 3D perovskites $^{7,26}$ and larger than the coupling strength in some inorganic quantum wells. ${ }^{37,38}$ 


\section{CONCLUSIONS}

In conclusion, we investigated the origin of the asymmetric $\mathrm{PL}$ spectra of self-assembled perovskite quantum wells $\left(\mathrm{BA}_{2} \mathrm{PbI}_{4}\right.$ and $\mathrm{HA}_{2} \mathrm{PbI}_{4}$ ) at room temperature. Using PL microscopy, we ruled out the probability of localized impurities causing the asymmetric emission. By investigating the PL emission on samples of different thickness, we confirmed that the asymmetric line shape is not due to reabsorption effects. When lowering the temperature, the $\mathrm{PL}$ shape of $\mathrm{HA}_{2} \mathrm{PbI}_{4}$ becomes narrower and symmetric, suggesting that the asymmetric PL shape at room temperature originates from exciton-phonon coupling. We performed femtosecond transient absorption spectroscopy to obtain a real-time picture of how excitons couple to phonons and directly identified the phonon modes that couple to excitons. It is found that changing the organic cations will affect the phonon modes of $2 \mathrm{D}$ perovskites. In $\mathrm{BA}_{2} \mathrm{PbI}_{4}$, excitons dominantly couple to phonons at $100 \mathrm{~cm}^{-1}$, whereas in $\mathrm{HA}_{2} \mathrm{PbI}_{4}$, excitons couple to phonons with frequencies of 88 and $137 \mathrm{~cm}^{-1}$. Based on the determined modes and a model that fits the temperaturedependent PL line width, we qualitatively studied the PL line width broadening mechanisms as a consequence of excitonphonon coupling. At low temperatures $(<100 \mathrm{~K})$, the line width broadening is due to acoustic phonon scattering at $0.026 \mathrm{meV} /$ $\mathrm{K}$, whereas at high temperatures, LO phonon-exciton coupling is the dominant mechanism that broadens the PL. In 2D perovskites, the LO phonon-exciton coupling strength is several tens of meV. Our results agree with what has been reported in 3D perovskites, ${ }^{7}$ where it is suggested that Fröhlich coupling between charge carriers and LO phonon modes dominates at room temperature.

Overall, strong exciton-phonon coupling in perovskite quantum wells may not be an advantage for LED devices because it causes line width broadening and may result in undesired asymmetric line shape. However, it is possible that the broadening can be suppressed by changing the organic ligands, as we show that the choice of organic ligands affects the exciton-phonon interaction. For other applications, strong exciton-phonon coupling in 2D perovskites can be a benefit; for instance, it can accelerate polariton relaxation and realize polariton lasing with very low threshold. ${ }^{39,40}$ It could also provide interesting opportunities for the development of broadband, short-pulsed lasers.

\section{METHODS}

Materials. All materials were purchased from Sigma-Aldrich or Dysol and were used as received. A solution of $\mathrm{PbI}_{2}$ and $\mathrm{CH}_{3}\left(\mathrm{CH}_{2}\right)_{3} \mathrm{NH}_{3} \mathrm{I}$ (or $\mathrm{CH}_{3}\left(\mathrm{CH}_{2}\right)_{5} \mathrm{NH}_{3} \mathrm{I}$ ) having a molar ratio of $1: 2$ was prepared in $\mathrm{N}, \mathrm{N}$-dimethylformamide solvent by constant stirring until dissolved. Inside a nitrogen-filled glovebox, the solution was then spin-coated on top of clean Spectrosil fused silica substrate at 2000 rpm for $60 \mathrm{~s}$ and annealed at $100{ }^{\circ} \mathrm{C}$ for $5 \mathrm{~min}$.

Absorption Measurement. Linear absorption spectra of thin films deposited on Spectrosil fused silica substrates were measured using a Hewlett-Packard 8453 UV-vis spectrometer with blank substrate correction.

Photoluminescence Measurement and Temperature-Dependent PL. For room temperature PL measurements, a CW 405 $\mathrm{nm}$ diode laser was used to photoexcite the samples. Emission was measured using an Andor iDus DU420A Si detector. The samples were encapsulated using glass coverslips to avoid degradation. For lowtemperature PL measurements, the samples were unencapsulated and placed in a continuous-flow cryostat (Oxford Instruments Optistat) under helium atmosphere. We allowed the sample temperature to equilibrate for $30 \mathrm{~min}$ before taking data. The excitation power was 4 $\mathrm{W} / \mathrm{cm}^{2}$.

Photoluminescence Microscopy. Microscopic photoluminescence mapping was performed using a WITec alpha 300 RAS confocal setup. The excitation source was a $405 \mathrm{~nm} \mathrm{CW}$ laser (Coherent CUBE). The laser was coupled through an optical fiber to the microscope and focused to a beam diameter about $0.5 \mu \mathrm{m}$ using a $100 \times$ objective $(\mathrm{NA}=0.9)$. The power output was $10 \mu \mathrm{W}$. Thin film samples were spin-coated on Spectrosil fused silica substrates and located on a $X-Y$ piezo stage under the objective. The PL spectra were collected in reflection mode with the same objective and acquired using a CCD detector. A long-pass filter with a cutoff wavelength of $435 \mathrm{~nm}$ was put before the detector to block the excitation beam. The movement of the stage was controlled by the WITec ScanCtr Spectroscopy Plus software, in which the PL maps were recorded.

Photothermal Deflection Spectroscopy. PDS is a scatter-free surface averaged absorption measurement capable of measuring 5-6 orders of magnitude weaker absorbance than the band-edge absorption. For the measurements, a monochromatic pump light beam produced by a combination of a Light Support MKII $100 \mathrm{~W}$ xenon arc source and a CVI DK240 monochromator is shone on the sample (film on Spectrosil fused silica substrate), inclined perpendicular to the plane of the sample, which on absorption produces a thermal gradient near the sample surface via nonradiative relaxation induced heating. This results in a refractive index gradient in the area surrounding the sample surface. This refractive index gradient is further enhanced by immersing the sample in an inert liquid FC-72 Fluorinert (3M Company) that has a high refractive index change per unit change in temperature. A fixed wavelength $\mathrm{CW}$ laser probe beam, produced using a Qioptiq $670 \mathrm{~nm}$ fiber-coupled diode laser with temperature stabilizer for reduced beam pointing noise, is passed through this refractive index gradient, producing a deflection proportional to the absorbed light at that particular wavelength, which is detected by a differentially amplified quadrant photodiode and lock-in amplifier (Stanford Research SR830) combination. Scanning through different wavelengths gives the complete absorption spectra.

Temperature-Dependent Absorption. Temperature-dependent absorption spectra were measured with an Agilent Cary 6000i UVvis-NIR spectrophotometer with blank substrate correction. Spincoated samples on fused silica substrates were unencapsulated and placed in a continuous-flow cryostat (Oxford Instruments Optistat) under helium atmosphere. We allowed the sample temperature to equilibrate for $30 \mathrm{~min}$ before taking data.

Steady-State Raman Spectroscopy. Raman measurements were conducted by backscattering (HORIBA T64000) at $633 \mathrm{~nm}$ (CW $\mathrm{HeNe}$ ) line with a subtractive triple stage. Spectra were collected between 26 and $650 \mathrm{~cm}^{-1}$ where the CCD detector (HORIBA Synapse Open-Electrode) has an approximately constant quantum efficiency $0.45 \pm 0.02$. Acquisitions employed a $100 \times$ optical objective and used minimal laser intensity to avoid laser damage.

Femtosecond Transient Absorption Spectroscopy. The fs-TA experiments were performed using an Yb-based amplified system (PHAROS, Light Conversion) providing $14.5 \mathrm{~W}$ at $1025 \mathrm{~nm}$ and 38 $\mathrm{kHz}$ repetition rate. The probe beam is generated by focusing a portion of the fundamental in a $4 \mathrm{~mm}$ YAG substrate and spans from 520 to $1400 \mathrm{~nm}$. The pump beam is generated in a noncollinear optical parametric amplifier (NOPAs; $37^{\circ}$ cut BBO, type I, $5^{\circ}$ external angle) pumped with the third harmonic of the source. The NOPA outputs $1 \mathrm{~mW}$ power centered at $520 \mathrm{~nm}$, with pulse durations of $16 \mathrm{fs}$ (upper limit determined by FROG). The white light is delayed using a computer-controlled piezoelectric translation stage, and a sequence of probe pulses with and without pump is generated using a chopper wheel on the pump beam. The pump irradiance was at $34 \mu \mathrm{J} / \mathrm{cm}^{2}$. After the sample, the probe pulse is seeded to a visible monochromator (550 $\mathrm{nm}$ blazed grating) and imaged using an InGaAs photodiode array camera (Sensors Unlimited). 


\section{ASSOCIATED CONTENT}

\section{S Supporting Information}

The Supporting Information is available free of charge on the ACS Publications website at DOI: 10.1021/acsnano.7b03984.

PDS, PL microscopy, PL on samples with different thickness, temperature-dependent PL and UV-vis, fsTA, and PL line width analysis results; measurement data (PDF)

\section{AUTHOR INFORMATION}

\section{Corresponding Author}

*E-mail: ar525@cam.ac.uk.

\section{ORCID}

Limeng Ni: 0000-0001-6604-7336

Aditya Sadhanala: 0000-0003-2832-4894

Akshay Rao: 0000-0003-4261-0766

Notes

The authors declare no competing financial interest.

Data related to this publication is available at the University of Cambridge data repository at https://doi.org/10.17863/CAM. 13802.

\section{ACKNOWLEDGMENTS}

This work was supported by the Engineering and Physical Sciences Research Council (EPSRC) and the Winton Programme for the Physics of Sustainability. L.N. acknowledges support from the Jardine Foundation. R.S. acknowledges a Newton-Bhabha international fellowship.

\section{REFERENCES}

(1) Saliba, M.; Matsui, T.; Seo, J.-Y.; Domanski, K.; Correa-Baena, J.P.; Nazeeruddin, M. K.; Zakeeruddin, S. M.; Tress, W.; Abate, A.; Hagfeldt, A.; Grätzel, M. Cesium-Containing Triple Cation Perovskite Solar Cells: Improved Stability, Reproducibility and High Efficiency. Energy Environ. Sci. 2016, 9, 1989-1997.

(2) Tsai, H.; Nie, W.; Blancon, J.-C.; Stoumpos, C. C.; Asadpour, R.; Harutyunyan, B.; Neukirch, A. J.; Verduzco, R.; Crochet, J. J.; Tretiak, S.; Pedesseau, L.; Even, J.; Alam, M. A.; Gupta, G.; Lou, J.; Ajayan, P. M.; Bedzyk, M. J.; Kanatzidis, M. G.; Mohite, A. D. High-Efficiency Two-Dimensional Ruddlesden-Popper Perovskite Solar Cells. Nature 2016, 536, 312-316.

(3) Smith, I. C.; Hoke, E. T.; Solis-Ibarra, D.; McGehee, M. D.; Karunadasa, H. I. A Layered Hybrid Perovskite Solar-Cell Absorber with Enhanced Moisture Stability. Angew. Chem., Int. Ed. 2014, 53, 11232-11235.

(4) Cao, D. H.; Stoumpos, C. C.; Farha, O. K.; Hupp, J. T.; Kanatzidis, M. G. 2D Homologous Perovskites as Light-Absorbing Materials for Solar Cell Applications. J. Am. Chem. Soc. 2015, 137, $7843-7850$.

(5) Yuan, M.; Quan, L. N.; Comin, R.; Walters, G.; Sabatini, R.; Voznyy, O.; Hoogland, S.; Zhao, Y.; Beauregard, E. M.; Kanjanaboos, P.; Lu, Z.; Kim, D. H.; Sargent, E. H. Perovskite Energy Funnels for Efficient Light-Emitting Diodes. Nat. Nanotechnol. 2016, 11, 872-877.

(6) Wang, N.; Cheng, L.; Ge, R.; Zhang, S.; Miao, Y.; Zou, W.; Yi, C.; Sun, Y.; Cao, Y.; Yang, R.; Wei, Y.; Guo, Q.; Ke, Y.; Yu, M.; Jin, Y.; Liu, Y.; Ding, Q.; Di, D.; Yang, L.; et al. Perovskite Light-Emitting Diodes Based on Solution-Processed Self-Organized Multiple Quantum Wells. Nat. Photonics 2016, 10, 699-704.

(7) Wright, A. D.; Verdi, C.; Milot, R. L.; Eperon, G. E.; PérezOsorio, M. A.; Snaith, H. J.; Giustino, F.; Johnston, M. B.; Herz, L. M. Electron-phonon Coupling in Hybrid Lead Halide Perovskites. Nat. Commun. 2016, 7, 11755.

(8) Zhu, H.; Miyata, K.; Fu, Y.; Wang, J.; Joshi, P. P.; Niesner, D.; Williams, K. W.; Jin, S.; Zhu, X.-Y. Screening in Crystalline Liquids
Protects Energetic Carriers in Hybrid Perovskites. Science 2016, 353, 1409-1413.

(9) Guo, Z.; Wu, X.; Zhu, T.; Zhu, X.; Huang, L. Electron-Phonon Scattering in Atomically Thin 2D Perovskites. ACS Nano 2016, 10, 9992-9998.

(10) Wu, X.; Trinh, M. T.; Niesner, D.; Zhu, H.; Norman, Z.; Owen, J. S.; Yaffe, O.; Kudisch, B. J.; Zhu, X. Y. Trap States in Lead Iodide Perovskites. J. Am. Chem. Soc. 2015, 137, 2089-2096.

(11) Dou, L.; Wong, A. B.; Yu, Y.; Lai, M.; Kornienko, N.; Eaton, S. W.; Fu, A.; Bischak, C. G.; Ma, J.; Ding, T.; Ginsberg, N. S.; Wang, L.W.; Alivisatos, A. P.; Yang, P. Atomically Thin Two-Dimensional Organic-Inorganic Hybrid Perovskites. Science 2015, 349, 1518-1521.

(12) Dohner, E. R.; Jaffe, A.; Bradshaw, L. R.; Karunadasa, H. I. Intrinsic White-Light Emission from Layered Hybrid Perovskites. J. Am. Chem. Soc. 2014, 136, 13154-13157.

(13) Yuan, Z.; Zhou, C.; Tian, Y.; Shu, Y.; Messier, J.; Wang, J. C.; van de Burgt, L. J.; Kountouriotis, K.; Xin, Y.; Holt, E.; Schanze, K.; Clark, R.; Siegrist, T.; Ma, B. One-Dimensional Organic Lead Halide Perovskites with Efficient Bluish White-Light Emission. Nat. Commun. 2017, 8, 14051

(14) De Wolf, S.; Holovsky, J.; Moon, S. J.; Löper, P.; Niesen, B.; Ledinsky, M.; Haug, F. J.; Yum, J. H.; Ballif, C. Organometallic Halide Perovskites: Sharp Optical Absorption Edge and Its Relation to Photovoltaic Performance. J. Phys. Chem. Lett. 2014, 5, 1035-1039.

(15) Sadhanala, A.; Deschler, F.; Thomas, T. H.; Dutton, S. E.; Goedel, K. C.; Hanusch, F. C.; Lai, M. L.; Steiner, U.; Bein, T.; Docampo, P.; Cahen, D.; Friend, R. H. Preparation of Single-Phase Films of $\mathrm{CH} 3 \mathrm{NH} 3 \mathrm{~Pb}(\mathrm{I} 1-\mathrm{xBrx}) 3$ with Sharp Optical Band Edges. J. Phys. Chem. Lett. 2014, 5, 2501-2505.

(16) Kronemeijer, A. J.; Pecunia, V.; Venkateshvaran, D.; Nikolka, M.; Sadhanala, A.; Moriarty, J.; Szumilo, M.; Sirringhaus, H. TwoDimensional Carrier Distribution in Top-Gate Polymer Field-Effect Transistors: Correlation between Width of Density of Localized States and Urbach Energy. Adv. Mater. 2014, 26, 728-733.

(17) Pathak, S.; Sepe, A.; Sadhanala, A.; Deschler, F.; Haghighirad, A.; Sakai, N.; Goedel, K. C.; Stranks, S. D.; Noel, N.; Price, M. Hüttner, S.; Hawkins, N. A.; Friend, R. H.; Steiner, U.; Snaith, H. J. Atmospheric Influence upon Crystallization and Electronic Disorder and Its Impact on the Photophysical Properties of Organic-Inorganic Perovskite Solar Cells. ACS Nano 2015, 9, 2311-2320.

(18) Diab, H.; Arnold, C.; Lédée, F.; Trippé-Allard, G.; Delport, G.; Vilar, C.; Bretenaker, F.; Barjon, J.; Lauret, J.-S.; Deleporte, E.; Garrot, D. Impact of Reabsorption on the Emission Spectra and Recombination Dynamics of Hybrid Perovskite Single Crystals. J. Phys. Chem. Lett. 2017, 8, 2977-2983.

(19) Yu, P. Y.; Cardona, M. Fundamentals of Semiconductors; Graduate Texts in Physics; Springer-Verlag, 2010.

(20) Billing, D. G.; Lemmerer, A. Synthesis, Characterization and Phase Transitions in the Inorganic-Organic Layered Perovskite-Type Hybrids $[(\mathrm{CnH} 2 \mathrm{n}+1 \mathrm{NH} 3)(2) \mathrm{PbI} 4], \mathrm{n}=4,5$ and 6. Acta Crystallogr., Sect. B: Struct. Sci. 2007, 63, 735-747.

(21) Ishihara, T.; Takahashi, J.; Goto, T. Optical Properties due to Electronic Transitions in Two-Dimensional Semiconductors $(\mathrm{CnH} 2 \mathrm{n}$ $+1 \mathrm{NH} 3) 2 \mathrm{PbI}$. Phys. Rev. B: Condens. Matter Mater. Phys. 1990, 42, 11099-11107.

(22) Yangui, A.; Sy, M.; Li, L.; Abid, Y.; Naumov, P.; Boukheddaden, K. Rapid and Robust Spatiotemporal Dynamics of the First-Order Phase Transition in Crystals of the Organic-Inorganic Perovskite (C12H25NH3)2PbI4. Sci. Rep. 2015, 5, 16634.

(23) Osherov, A.; Hutter, E. M.; Galkowski, K.; Brenes, R.; Maude, D. K.; Nicholas, R. J.; Plochocka, P.; Bulović, V.; Savenije, T. J.; Stranks, S. D. The Impact of Phase Retention on the Structural and Optoelectronic Properties of Metal Halide Perovskites. Adv. Mater. 2016, 28, 10757-10763.

(24) Hu, T.; Smith, M. D.; Dohner, E. R.; Sher, M. J.; Wu, X.; Trinh, M. T.; Fisher, A.; Corbett, J.; Zhu, X. Y.; Karunadasa, H. I.; Lindenberg, A. M. Mechanism for Broadband White-Light Emission from Two-Dimensional (110) Hybrid Perovskites. J. Phys. Chem. Lett. 2016, 7, 2258-2263. 
(25) Gauthron, K.; Lauret, J.; Doyennette, L.; Lanty, G.; Al Choueiry, A.; Zhang, S. J.; Brehier, A.; Largeau, L.; Mauguin, O.; Bloch, J.; Deleporte, E. Optical Spectroscopy of Two-Dimensional Layered (C6H5C2H4-NH3)2-PbI4 Perovskite. Opt. Express 2010, 18, 59125919.

(26) Fang, H.; Wang, F.; Adjokatse, S.; Zhao, N.; Even, J.; Loi, M. A. Photoexcitation Dynamics in Solution- Processed Formamidinium Lead Iodide Perovskite Thin Films for Solar Cell Applications Running Title: Optical Properties of a Hybrid Perovskite. Light: Sci. Appl. 2016, 5, e16056-7.

(27) Musser, A. J.; Liebel, M.; Schnedermann, C.; Wende, T.; Kehoe, T. B.; Rao, A.; Kukura, P. Evidence for Conical Intersection Dynamics Mediating Ultrafast Singlet Exciton Fission. Nat. Phys. 2015, 11, 352357.

(28) Schnedermann, C.; Liebel, M.; Kukura, P. Mode-Specificity of Vibrationally Coherent Internal Conversion in Rhodopsin during the Primary Visual Event. J. Am. Chem. Soc. 2015, 137, 2886-2891.

(29) Pérez-Osorio, M. A.; Milot, R. L.; Filip, M. R.; Patel, J. B.; Herz, L. M.; Johnston, M. B.; Giustino, F. Vibrational Properties of the Organic-Inorganic Halide Perovskite $\mathrm{CH} 3 \mathrm{NH} 3 \mathrm{PbI} 3$ from Theory and Experiment: Factor Group Analysis, First-Principles Calculations, and Low-Temperature Infrared Spectra. J. Phys. Chem. C 2015, 119, 25703-25718.

(30) Lee, J.; Koteles, E. S.; Vassell, M. O. Luminescence Linewidths of Excitons in GaAs Quantum Wells below $150 \mathrm{~K}$. Phys. Rev. B: Condens. Matter Mater. Phys. 1986, 33, 5512-5516.

(31) Rudin, S.; Reinecke, T. L. Temperature-Dependent Exciton Linewidths in Semiconductor Quantum Wells. Phys. Rev. B: Condens. Matter Mater. Phys. 1990, 41, 3017-3027.

(32) Chen, Y.; Kothiyal, G. P.; Singh, J.; Bhattacharya, P. K. Absorption and Photoluminescence Studies of the Temperature Dependence of Exciton Life Time in Lattice-Matched and Strained Quantum Well Systems. Superlattices Microstruct. 1987, 3, 657-664.

(33) Leroux, M.; Grandjean, N.; Beaumont, B.; Nataf, G.; Semond, F.; Massies, J.; Gibart, P. Temperature Quenching of Photoluminescence Intensities in Undoped and Doped GaN. J. Appl. Phys. 1999, 86, 3721.

(34) Akasaka, T.; Gotoh, H.; Saito, T.; Makimoto, T. High Luminescent Efficiency of InGaN Multiple Quantum Wells Grown on InGaN Underlying Layers. Appl. Phys. Lett. 2004, 85, 3089-3091.

(35) Jiang, D. S.; Jung, H.; Ploog, K. Temperature Dependence of Photoluminescence from GaAs Single and Multiple Quantum-Well Heterostructures Grown by Molecular-Beam Epitaxy. J. Appl. Phys. 1988, 64, 1371-1377.

(36) Rudin, S.; Reinecke, T. L. Exciton-Acoustic-Phonon Linewidths in GaAs Bulk and Quantum Wells. Phys. Rev. B: Condens. Matter Mater. Phys. 2002, 65, 121311.

(37) Shen, W. Z.; Shen, S. C.; Tang, W. G.; Wang, S. M.; Andersson, T. G. Observation of Excitonic Polariton and Broadening of RoomTemperature Exciton in Strained InGaAs/GaAs Quantum Wells. J. Appl. Phys. 1995, 78, 1178-1182.

(38) O’Neill, M.; Oestreich, M.; Rühle, W. W.; Ashenford, D. E. Exciton Radiative Decay and Homogeneous Broadening in CdTe/ Cd0.85Mn0.15Te Multiple Quantum Wells. Phys. Rev. B: Condens. Matter Mater. Phys. 1993, 48, 8980-8985.

(39) Orosz, L.; Réveret, F.; Médard, F.; Disseix, P.; Leymarie, J.; Mihailovic, M.; Solnyshkov, D.; Malpuech, G.; Zuniga-Pérez, J.; Semond, F.; Leroux, M.; Bouchoule, S.; Lafosse, X.; Mexis, M.; Brimont, C.; Guillet, T. LO-Phonon-Assisted Polariton Lasing in a ZnO-Based Microcavity. Phys. Rev. B: Condens. Matter Mater. Phys. 2012, 85, 121201.

(40) Maragkou, M.; Grundy, A. J. D.; Ostatnicky, T.; Lagoudakis, P. G. Longitudinal Optical Phonon Assisted Polariton Laser. Appl. Phys. Lett. 2010, 97, 111110. 